\title{
Die österreichische Scheidungsreform in den 1970er Jahren
}

In Austria during the 1970s extensive reforms in the area of family law were passed. These reforms were adopted unanimously in parliament with one exception. The reform of divorce law, which was carried out in 1978 by the implementation of two new laws, only partly was decided by common agreement. While the new possibility of divorce by consent was introduced with the votes of all parties, the new regulations on divorce against the will of the blameless spouse, led not only to the end of consent in parliament. It also evoked protest of the Catholic Church and the Social Democratic Women. The article provides an overview on the historical development of divorce law in Austria until 1945 and the reform process in the Second Republic with a focus on the social and political negotiations in the 1970s.

Die 1970er Jahre markieren in Österreich ein Jahrzehnt, in dem große Reformen - sowohl im Bereich des Straf-, als auch des Familienrechts verabschiedet wurden. Betrachtet man diese ganz allgemein, fällt auf, dass sie mit wenigen Ausnahmen einstimmig verabschiedet wurden. Zum Scheitern kam der Konsensmobilisierungsprozess lediglich in zwei Bereichen: in der Strafrechtsreform bei der Einführung der Fristenregelung und in der Familienrechtsrechtform bei der Scheidungsreform. Das neue Scheidungsrecht stellt somit nach der Neuregelung der Rechtsstellung des unehelichen Kindes (1970), den persönlichen Rechtswirkungen der Ehe (1975), der Einführung des Unterhaltsvorschussgesetzes (1976) und Reformen in der Rechtsstellung des ehelichen Kindes (1977) mit dem neuen Ehegattenerb- und -güterrecht (1978) nicht nur den Schlusspunkt der Familienrechtsreform dar. Es markiert auch ihr "heißes Eisen“ und hat das von der SPÖ geführte Justizministerium nicht nur in Konflikt mit der ÖVP, sondern auch mit der katholischen Kirche und Teilen ihrer Frauenorganisation gebracht.
Im Folgenden soll in Form eines knappen Rückblicks zunächst die historische Entwicklung des Scheidungsrechts in Österreich skizziert werden, bevor näher auf die Reformdiskussion nach 1945 eingegangen wird. Deutlich wird hierbei, dass das Scheidungsrecht bereits in früheren Jahren eine stark umkämpfte Materie war.

\section{Scheidungsrecht im ABGB bis 1938}

Das ABGB kannte ein dreifach gegliedertes Eherecht für Katholiken, Protestanten und Juden und unterschied in eine "Scheidung von Tisch und Bett" und „Trennung“, wobei nur die Trennung die gänzliche Auflösung der Ehe bewirkte. Dabei galt, dass Ehen, die Katholiken vor einem Priester zu schließen hatten, im Gegensatz zu Ehen Andersgläubiger nicht vollständig auflösbar waren bzw. für sie nur eine „Scheidung von Tisch und Bett" ohne anschließende Wiederverehelichung möglich war. Bestrebungen dies zu ändern, reichen zwar bereits bis 19. Jahrhundert zurück, konnten in Österreich im Vergleich zu 
anderen Staaten jedoch nicht verwirklicht werden, da das Eherecht unter einem deutlichen Einfluss der katholischen Kirche stand. ${ }^{1}$

Nach der Ausrufung der Republik wurde die Forderung nach der Einführung der Zivilehe und damit verbunden auch nach der Trennbarkeit der Katholikenehen zu einem zentralen Streitthema zwischen den Parteien. Hierbei setzten sich vor allem die Sozialdemokraten für die Einführung der Zivilehe ein, während die Christlichsozialen auch nach dem Untergang der Monarchie zum zentralen Bündnispartner der katholischen Kirche wurden. Mehrere Anträge, die im Parlament eingebracht wurden, fanden aufgrund der politischen Machtverhältnisse und weltanschaulichen Differenzen keine Beschlussfassung. Nicht zuletzt deshalb wurde auch auf administrativem Weg versucht, das Problem zu lösen, womit die so genannten "Dispensehen" angesprochen werden. Maßgeblich ist hierbei, dass eine unklare Bestimmung im ABGB hinsichtlich des landesfürstlichen Gnadenrechts dazu genutzt wurde, nach einer "Scheidung von Tisch und Bett" vom Ehehindernis des aufrechten Ehebandes zu dispensieren, wonach die Ehewerber vor einer staatlichen Behörde im Weg einer Notzivilehe oder nach einem Religionswechsel vor einem nichtkatholischen Seelsorger eine zweite Ehe schließen konnten. Über die Gültigkeit dieser Ehen fand in Folge jedoch ein Jahre langer Rechtsstreit statt. ${ }^{2}$

\section{Ehegesetz 1938}

Eine entscheidende Änderung brachte erst das neue EheG von 6. Juli 1938, das einerseits das in Österreich herrschende Chaos auf dem Gebiet des Eherechts beseitigen und andererseits auch die Ehen der Österreicher dem Zugriff des NS-

\footnotetext{
${ }^{1}$ SCHAUER, Recht der Ehescheidung 42-67.

${ }^{2}$ HARMAT, Ehe auf Widerruf?
}

Staates unterwerfen sollte. ${ }^{3}$ Ausgehend davon, dass die Bedeutung von Ehe und Familie für die Nationalsozialisten in erster Linie in der Weitergabe "rassisch wertvollen Erbguts" lag, brachte es zum einen den Einzug der NS-Rassenideologie ins Eherecht, zugleich führte es aber auch die obligatorische Zivilehe, wie sie im Deutschen Reich 1875 geschaffen und 1896 ins Bürgerliche Gesetzbuch übernommen worden war, sowie ein für die Angehörigen aller Religionsgemeinschaften gleiches Ehescheidungsrecht ein. Dieses ermöglichte nun auch für Katholiken in Österreich eine Trennung der Ehe mit anschließender Wiederverehelichung, wobei seither der Terminus "Scheidung" auch in Österreich für eine vollständige Auflösung der Ehe verwendet wird.

Was die konkreten Scheidungsgründe betrifft, sah das EheG 1938 eine Kombination aus Verschuldens- und Zerrüttungsprinzip vor. Subsumiert wurden unter "Scheidung wegen Verschuldens" Ehebruch, Verweigerung der Fortpflanzung und andere Eheverfehlungen, während die auf einer Zerrüttung beruhenden „anderen Scheidungsgründe" neben auf geistiger Störung beruhendem Verhalten, Geisteskrankheit, ansteckender oder Eckel erregender Krankheit und Unfruchtbarkeit auch die Auflösung der häuslichen Gemeinschaft (§55 EheG) umfasste. ${ }^{4}$ Dass sich die nationalsozialistische Politik für das Zerrüttungsprinzip erwärmen konnte, ursprünglich sogar als einzigen Scheidungsgrund erwog, entsprach jedoch keineswegs einer individualisierenden Ehevorstellung, wie sie in der Weimarer Republik diskutiert worden war. Vielmehr stand dahinter - in Kombination mit dem generalklauselartig, aber unbestimmt definierten Begriff vom „Wesen der

\footnotetext{
${ }^{3}$ Zusätzlich zu den geschilderten Problemen bestand auch noch jener Sonderfall, dass im ehemals zu Ungarn gehörenden Burgenland die obligatorische Zivilehe galt.

${ }^{4}$ LEHNER, Familie - Recht - Politik 149-183.
} 
Ehe" - die Absicht, die "richtige Ehe“ von Staats wegen $\mathrm{zu}$ kontrollieren und Scheidungen in einem größeren Maß zu ermöglichen, wenn aus einer zweiten Ehe eine Steigerung der Bevölkerungsrate zu erwarten war. ${ }^{5}$ Dem entsprach, dass es im Scheidungsfolgenrecht auch zu einer erheblichen finanziellen Verschlechterung des ersten Ehegatten kommen konnte. Eine einvernehmliche Auflösung der Ehe, die es im ABGB sowohl hinsichtlich der Trennung von Protestanten und Juden als auch der "Scheidung von Tisch und Bett" bei den Katholiken gegeben hatte, sah das EheG 1938 jedoch nicht vor, da dies ein liberales Element bedeutet hätte. ${ }^{6}$

\section{Reformdiskussion nach 1945}

1945 wurde das EheG 1938 mit einigen Ausnahmen, in denen „typisches Gedankengut des Nationalsozialismus" gesehen wurde, von der Republik Österreich übernommen. Das Scheidungsrecht des EheG 1938 galt mit Ausnahme der Scheidung wegen Unfruchtbarkeit auch nach 1945 weiter. $^{7}$

Betrachtet man nun die Ehe- und Familienrechtsdiskussion in der Zweiten Republik, fällt einerseits auf, dass zwischen den Parteien recht bald wieder eine Diskussion über die obligatorische Zivilehe geführt wurde. ${ }^{8}$ Andererseits ist beachtlich, dass das Scheidungsrecht auf Seiten des Justizministeriums in seinen Reformüberlegungen lange ausgeklammert wurde, während sich die Rechtswissenschaft des Themas bald

\footnotetext{
${ }^{5}$ GRUCHMANN, Ehegesetz.

${ }^{6}$ LEHNER, Familie - Recht - Politik 162-166.

${ }^{7}$ Im Gegensatz zur BRD blieb die Verweigerung der Nachkommenschaft als Scheidungsgrund in Kraft. Mit dem EheRÄG 1999 wurden die Scheidungsgründe mit absoluter Wirkung (Ehebruch, Verweigerung der Fortpflanzung) beseitigt und in einen einzigen relativen Verschuldensscheidungstatbestand integriert. Vgl. SCHAUER, Gesunde Nachkommenschaft.

${ }^{8}$ LEHNER, Familie - Recht - Politik 218-222.
}

wieder annahm. Angesprochen - und das äußerst kontrovers - wurden von Seiten der Fachwelt eine Reihe von Punkten - darunter die Einführung der Konventionalscheidung, da sich hinter den meisten wegen $\S 49$ EheG (andere Eheverfehlungen) geschiedenen Ehen eigentlich einvernehmliche Scheidungen verbargen, sowie eine Neuregelung der Scheidung wegen der Auflösung der ehelichen Gemeinschaft. Wesentlich wurde hierbei insbesondere, dass es von Seiten der Judikatur in den Jahren nach $1945 \mathrm{zu}$ einer völligen Umkehr in der Auslegung des $\S 55$ EheG kam. Nach diesem konnte jeder Ehegatte die Scheidung infolge einer tiefgreifenden unheilbaren Zerrüttung beantragen, wenn die Wiederherstellung einer dem Wesen der Ehe entsprechenden Lebensgemeinschaft nicht $\mathrm{zu}$ erwarten war und die Aufhebung der häuslichen Gemeinschaft drei Jahren gedauert hatte. Hatte der Ehegatte, der die Scheidung begehrte, die Zerrüttung jedoch ganz oder überwiegend verschuldet, konnte der andere Ehegatte einen Widerspruch erheben, worauf das Gericht zu entscheiden hatte, ob dieser Widerspruch beachtlich sei oder nicht. Während dem Widerspruch vor 1945 nur selten Beachtung eingeräumt wurde, da als Meta-Regel die Abwägung diente, was dem Staat mehr nützt - die Aufrechterhaltung der alten Ehe oder die Gründung einer Neuen - wurde der Widerspruch nach 1945 jedoch zumeist für beachtlich erklärt. Motiviert war dies im Sinn einer geänderten Wertvorstellung der Ehe und einer Betonung der Beistandspflicht insbesondere dadurch, dass die Scheidung eine Verschlechterung im Unterhalt des schuldlos geschiedenen Ehegatten, in der Regel die Frau, bringen würde. ${ }^{9}$

Die politischen Parteien und auch das Justizministerium waren sich dieser Problematik zwar bewusst, wegen ihrer gesellschaftspolitischen Brisanz wurde diese Frage jedoch lange zurück-

\footnotetext{
${ }^{9}$ Ebd. 516-520.
} 
gestellt. So war das Scheidungsrecht etwa kein Thema in der von SPÖ-Justizminister Otto Tschadek 1949 eingesetzten Familienrechtskommission, und auch das SPÖJustizprogramm von 1969, das die programmatische Grundlage für die Rechtsreform in der SPÖ-Alleinregierung ab 1970 darstellte, ${ }^{10}$ umfasste eine Reform des Scheidungsrechts ebenso wenig wie die ersten beiden Regierungserklärungen der Ära Kreisky. Verwiesen wurde hier lediglich allgemein auf einen Ausbau der unterhaltsrechtlichen Stellung des schutzbedürftigen Ehegatten für den Fall der Ehescheidung sowie darauf, dass der Ehefrau (bei Tod oder Scheidung) im Rahmen einer Neuregelung des Ehegüterrechts ein Anteil am Ehegewinn zukommen soll. Genannt wurde eine Reform des Scheidungsrechts erstmals in der Regierungserklärung vom 5. November 1975. In dieser hieß es, dass unter der Voraussetzung einer befriedigenden Lösung der unterhalts- und pensionsrechtlichen Absicherung der schutzbedürftigen Ehefrau auch einer „zeitgemäßen Anpassung des Scheidungsrechts (Novellierung des §55 EheG)" nähergetreten werden kann. ${ }^{11}$

Ein erstes Bekenntnis zur Scheidungsreform war von Justizminister Christian Broda indessen bereits 1970 in einem Beitrag in der "Zukunft", dem theoretischen Organ der SPÖ, abgegeben worden. ${ }^{12}$ Weitere Impulse erhielt die Scheidungsreform in Folge aus dem Ausland, vom österreichischen Anwaltstag $1972^{13}$ und einem Erkenntnis des OGH vom 25. Oktober 1972.14 Dieses enttäuschte nicht nur bestehende Hoffnungen nach einem Wandel in der Auslegung

\footnotetext{
${ }^{10}$ Mehr Rechtsschutz für den Staatsbürger. Justizprogramm für 1970-1974. Beschlossen vom Parteirat der SPÖ am 22. 11. 1969.

11 Gottschlich, Panagl, Welan, Was die Kanzler sagten.

12 BRODA, Ein „offenes“ Programm 18-20.

${ }^{13}$ Sondernummer des Österreichischen Anwaltsblatts vom November 1972.

${ }^{14}$ AChB, ÖNB, HS, V.100
}

des $\S 55$ EheG, sondern hielt vielmehr in Reaktion auf die andauernde Kritik fest, dass „unbefriedigende Gesetzesbestimmungen zu ändern nicht die Sache der Rechtsprechung, sondern der Gesetzgebung“ sei. Neuerlich wiederholt wurde dieser Auftrag an den Gesetzgeber im Tätigkeitsbericht des OGH 1973, wobei er gleichzeitig dahin gehend präzisiert wurde, eine zu Lösung finden, die den schuldlosen Teil vor einem Verlust seiner bisherigen Lebensgrundlage sichert. ${ }^{15}$

\section{Vom Ministerialentwurf 1976 zur Reform 1978}

Bis ein erster Ministerialentwurf fertig gestellt und zur Begutachtung ausgesendet werden konnte, dauerte es jedoch noch bis Anfang Jänner 1976. Hierbei galt es für das Justizministerium zuerst einmal abzustecken, welche Bereiche eine Scheidungsreform umfassen konnte, insbesondere, ob eine "große" oder eine „kleine Scheidungsreform" angestrebt werden soll. Während von Seiten des Ministeriums Überlegungen zu einer grundlegenden Reform des Scheidungsrechts angestellt wurden, ${ }^{16}$ entschied Broda jedoch relativ rasch, dass eine Reform vorbereitet werden soll, in der die textlichen Änderungen so klein wie möglich gehalten werden. Dementsprechend hieß es bereits im September 1975 in der dritten Auflage der Broschüre „Familienrechtsreform konkret“, mit der das Ministerium kontinuierlich seine Reformvorhaben begleitete, dass "daran gedacht [wird], vorerst mit einer ,Kleinen Scheidungsreform' zu beginnen", die einerseits eine Scheidung auch gegen den Widerstand des Ehepartners bringen soll und in der "gleich auf welchen Scheidungsgrund die Scheidung gestützt wird" auch dafür Vorsorge getroffen wird, dass es zu keiner Ver-

\footnotetext{
${ }^{15}$ Ebd.

${ }^{16}$ LEHNER, Familie - Recht - Politik 528-530.
} 
schlechterung für den an der Scheidung unschuldigen Teil kommen soll. „Eine darüber hinausgehende Reform durch eine grundlegende Neuordnung der Scheidungsgründe, so besonders durch Einführung der Möglichkeit einer sogenannten ,einverständlichen Scheidung' und durch Ersetzung des ,Verschuldensprinzips' durch das ,Zerrüttungsprinzip', der Scheidungsfolgen ganz allgemein und des Scheidungsverfahrens" sollte „einer späteren ,Großen Scheidungsreform ' vorbehalten werden."17 Hintergrund hierfür war einerseits die Erwägung, die Scheidungsreform zwischen die anderen Familienrechtsreformgesetze einzuschieben, anderseits spielten Befürchtungen, dass die Widerstände bei der Reform des Scheidungsrechts besonders groß sein würden, eine Rolle. Erwartet wurden diese nicht nur von der katholischen Kirche, sondern auch von den Frauen.

In Folge wurde eine Reform vorbereitet, die sich lediglich auf eine Änderung des $\S 55$ EheG bezog und eine Beseitigung des Widerspruchs nach einer fünfjährigen Aufhebung der häuslichen Gemeinschaft vorsah. Gelöst werden sollte damit das Problem der so genannten „Papierehen", sprich jener Ehen, die nur mehr auf dem Papier bestanden. Hinsichtlich der Scheidungsfolgen wurde zunächst an die Schaffung einer Fiktionslösung gedacht, die eine unterhalts- und sozialversicherungsrechtliche Stellung wie bei aufrechter Ehe - und dies nicht nur für Scheidungen nach $\S 55$ EheG - bringen sollte. Später wurde aus Gründen der Finanzierbarkeit jedoch von einer solchen Lösung abgegangen und die Reform des unterhalts- und versorgungsrechtlichen Scheidungsfolgenrechts nur mehr auf den $\S 55$ EheG bezogen. Ausgeführt wurde im Ministerialentwurf, dass sich der Unterhaltsanspruch des beklagten Ehegatten bei einer Scheidung nach $\S 55$ EheG so wie in aufrechter Ehe

\footnotetext{
${ }^{17}$ Bundesministerium für Justiz, Familienrechtsreform konkret $127 f$.
}

nach dem EheRwG 1975 bestimmen soll, sofern das Gericht dies in dem auf Scheidung lautenden Urteil festhielt. Dieses wiederum hatte eine solche Feststellung zu treffen, wenn der beklagte Ehegatte dies beantragt und der Gatte, der die Scheidung begehrt, die Zerrüttung ganz oder überwiegend verschuldet hatte. Was hingegen die sozialversicherungsrechtlichen Aspekte der Scheidung betrifft, die auf Druck der SPÖFrauen in die Verhandlungen einbezogen worden waren, sollte nach dem Abgehen von der Fiktionslösung vom Sozialministerium eine Novellierung der entsprechenden Gesetze vorbereitet werden.

Die ÖVP präsentierte ihre Vorstellungen zur Scheidungsreform wenige Tage nach der Versendung des Ministerialentwurfs. Besonders interessant ist hierbei ein Kommentar ihres Justizsprechers Walter Hauser, wonach diese Vorschläge bereits in der Zeit der ÖVPAlleinregierung Klaus ausgearbeitet, aber damals parteiintern nicht durchsetzbar waren. ${ }^{18}$ Eingetreten ist die ÖVP in ihren Vorschlägen für eine maßvolle Weiterentwicklung des geltenden Scheidungsrechts auf Basis der Beibehaltung eines Mischsystems aus Verschuldens- und Zerrüttungsprinzip. Zugleich votierte sie dafür, dass sich die Scheidungsreform nicht nur auf eine Änderung des $\S 55$ EheG beschränken soll. So forderte sie auch die Einführung einer einvernehmlichen Scheidung, allerdings unter folgenden - wie festgehalten wurde - „strengen einschränkenden Voraussetzungen": eine gewisse Mindestdauer der Ehe, eine mindestens einjährige Aufhebung der häuslichen Gemeinschaft, eine Einigung der Ehegatten über die Scheidungsfolgen, eine Genehmigung dieser Einigung durch das Gericht sowie die Möglichkeit, das Verfahren für die Dauer von sechs Monaten von Gerichts wegen auszusetzen, wenn es

\footnotetext{
${ }^{18}$ HAUSER: Scheidungsreform kein Vorschub für Sozialismus, in: Kleine Zeitung, 30. 3. 1976.
} 
zu der Überzeugung kam, dass eine Versöhnung der Eheleute möglich ist. Hinsichtlich der Änderung des §55 EheG hielt die ÖVP zwar ihre Zustimmung dazu fest, dass die von der Judikatur entwickelte grundsätzliche Beachtlichkeit des Widerspruchs fallen soll. Allerdings bestand sie - wie sich bereits in überparteilichen Gesprächen an der Jahreswende 1974/75 gezeigt hatte - auf der Einführung einer zeitlich unbefristeten immateriellen Härteklausel. Wirtschaftliche Umstände sollten jedoch außer Betracht bleiben. In der Frage des Unterhaltsanspruchs bei einer Scheidung nach $\S 55$ EheG votierte sie für einen klaren Vorrang für den ersten Ehegatten und kritisierte am Broda-Vorschlag, dass dieser einen Unterhaltsanspruch "wie in der Ehe" nur vortäusche. Eine Besserstellung gegenüber Scheidungen aufgrund anderer Scheidungsgründe sei gerechtfertigt, da bei Scheidungen nach $\S 55$ EheG der schuldlose Eheteil gegen seinen Willen geschieden wird, während in den anderen Fällen der unterhaltsberechtigte Ehegatte selbst der klagende Eheteil sei. Zugleich forderte sie hinsichtlich des weiteren Fahrplans in der Familienrechtsreform, dass das neue Scheidungsrecht nicht vor der damals noch offenen Neuregelung des Kindschaftsrechts sowie dem neuen Ehegüter- und -erbrecht verhandelt werden soll, womit sie sich dem Plan Brodas, die Scheidungsreform zwischendurch einzuschieben, widersetzte. ${ }^{19}$

Der Katholische Familienverband und der österreichische Laienrat, die sich intensiv in die Diskussion um ein neues Scheidungsrecht einbrachten, präsentierten ihr Gegenmodell zur Scheidungsreform im April 1976. Hierin stimmten sie unter acht (gegenüber dem ÖVP-Vorschlag verschärften) Bedingungen einer Scheidung im außerstreitigen Verfahren zu und brachten auch einen Alternativentwurf zur Neugestaltung des

${ }^{19}$ ÖVP-Zielsetzungen zur Reform des Scheidungsrechtes vom 22. 1. 1976, in: LEHNER, Familie - Recht Politik 649.
$\S 55$ EheG ein, der dem Richter die Möglichkeit geben sollte abzuwägen, ob die Aufrechterhaltung der Ehe den klagenden Gatten unverhältnismäßig härter träfe als die Scheidung den anderen. Dabei sollte bei der Abwägung auf alle Umstände des Einzelfalles, besonders auf die Dauer der Ehe, die Ursachen der Zerrüttung, das Wohl der Kinder, das Alter der Ehegatten Bedacht genommen werden. Eine „Fristenautomatik" wie sie der Ministerialentwurf vorgesehen hat, wurde jedoch schärfstens abgelehnt. ${ }^{20}$ Von Kardinal König war indessen bereits einige Wochen zuvor erklärt worden, dass es von Seiten der katholischen Kirche kein Veto gegen die geplante Scheidungsreform geben werde. Die katholische Kirche bekenne sich zwar zur Unauflöslichkeit der Ehe. In der pluralistischen Gesellschaft verlange sie jedoch nicht, dass der Staat ihre Ordnung des Eherechts denen vorschreibt, die die katholische Sicht der Ehe ablehnen. Dafür, dass vermeidbare Gegensätze zwischen staatlicher Rechtsordnung und kirchlicher Auffassung unterbleiben, wollte sie sich jedoch einsetzen. ${ }^{21}$

In der am 29. Juni 1976 im Nationalrat eingebrachten Regierungsvorlage wurde von Seiten des Justizministers zur Unterstützung seines Reformvorhabens nicht nur darauf verwiesen, das Reformen mit ähnlichen Zielsetzungen in den letzten Jahren auch in anderen europäischen Ländern durchgeführt worden waren, sondern zumindest teilweise auch auf die Vorschläge der ÖVP eingegangen. Neu war, dass die Regierungsvorlage nun auch die Einführung einer einvernehmlichen Scheidung - allerdings unter weniger strengen Voraussetzungen (ohne Vereinbarung über die Scheidungsfolgen und die Möglichkeit, das Verfahren für sechs Monate aussetzen zu können) - vorsah, wenngleich

\footnotetext{
${ }^{20}$ Katholischer Familienverband Österreichs, Neues Familienrecht 53-55.

${ }^{21}$ Kein Veto gegen geplante Scheidungsreform, in: Kronen Zeitung, 2. 2. 1976.
} 
Broda auch davor warnte, dass durch sie keine Verzögerung der überfälligen Teilreform des $\S 55$ EheG eintreten dürfe. ${ }^{22}$ Hinsichtlich der Scheidung wegen der Aufhebung der häuslichen Gemeinschaft wurde nun eine Formulierung gewählt, wonach der Widerspruch nur dann zu beachten sei, wenn die Scheidung für den Beklagten eine außergewöhnliche Härte darstellt. Allerdings sollte dessen Zulässigkeit mit dem vollendeten fünften Jahr der Aufhebung der häuslichen Gemeinschaft enden, womit am Kern der Reform festgehalten worden war. Bezüglich des Unterhalts wurde eine komplizierte Regelung vorgeschlagen, nach der der an der Zerrüttung schuldlose Ehegatte auf Antrag auch nach der Scheidung einen Anspruch nach $\S 94$ ABGB in der Fassung des EheRwG vom 1. Juli 1975 haben sollte. Der Unterhaltsanspruch des neuen Ehegatten war bei der Berechnung nicht zur berücksichtigen, trafen bestimmte Kriterien $\mathrm{zu}$, musste sich der geschiedene Ehegatte aber eine unterhaltsrechtliche Schlechterstellung gefallen lassen. Hinsichtlich der sozialversicherungsrechtlichen Scheidungsfolgen wurde - nachdem bereits Anfang 1976 ein erster Entwurf vorgelegt worden war $^{23}$ - in einer weiteren Regierungsvorlage des Sozialministeriums (neben Änderungen in der Krankenversicherung) Folgendes vorgesehen: die geschiedene Frau sollte die Witwenpension oder -rente in der vollen Höhe wie die Witwe erhalten, wenn die Ehe mindestens 15 Jahre gedauert und sie im Zeitpunkt der Einbringung der Ehescheidungsklage das 40. Lebensjahr vollendet hatte bzw. falls sie dieses Alter noch nicht erreicht hatte in der Ehe ein Kind geboren worden war, das

\footnotetext{
${ }^{22}$ Vgl. etwa: Rede von Christian Broda vor dem SPÖBundesfrauenkomitee am 24.1.1976. AChB, ÖNB, HS, V.113.1.

${ }^{23}$ Bundesministerium für Justiz, Scheidungsreform konkret 39-46.
}

bei Rechtskraft des Scheidungsurteils noch nicht zehn Jahre alt war. ${ }^{24}$

Die Reaktionen auf die Änderung des EheG drehten sich - wie zu erwarten war - vor allem um zwei Punkte: die Befristung des Widerspruchs und die unterhaltsrechtliche Stellung der Frau, womit Konflikte mit der großen Oppositionspartei und der katholischen Kirche vorprogrammiert waren. Besonders schwer wog für die SPÖ jedoch, dass es im Herbst $1977 \mathrm{zu}$ einer öffentlich geführten Auseinandersetzung mit Teilen der SPÖ-Frauen kam. Streitpunkt der Kontroverse, die ihren Höhepunkt in einem Interview der steirischen SPÖ-Frauenvorsitzenden Jolanda Offenbeck in der „Neuen Zeit" am 29. September 1977 und der SPÖKlubklausur im Oktober 1977 in Villach fand, war jedoch nicht die Befristung des Widerspruchsrechts. Vielmehr wurde die fehlende Vorrangstellung der ersten Frau im Unterhaltsrecht kritisiert und festgehalten, dass der nun vorliegende Vorschlag nicht der Regierungserklärung 1975 entspreche. Besondere Betonung fand hierbei auch der Hinweis, dass Broda die Initiative zur Scheidungsreform ergriffen habe, als der innerparteiliche Diskussionsprozess noch nicht abgeschlossen war. ${ }^{25}$ Um der innerparteilichen Kritik zu begegnen, wurde in Folge noch im November 1977 ein neuer Vorschlag ausgearbeitet, der die Stellung der ersten Frau im Unterhaltsrecht verbesserte. Zuvor hatte auch Bundeskanzler Kreisky festgehalten, dass man primär an die erste Frau denken müsse, die zweite Frau aber nicht vergessen dürfe. ${ }^{26}$ Ausge-

\footnotetext{
${ }^{24}$ Da die Neuregelung des $\S 55$ EheG geschlechtsneutral formuliert wurde, war erstmals (unter ähnlichen Voraussetzungen) auch für geschiedene Witwer eine Hinterbliebenenpension vorgesehen, solange sie erwerbsunfähig und bedürftig waren.

${ }^{25}$ Offenbeck: Bei der Unterhaltsregelung mehr Rücksicht auf die erste Frau nehmen, in: Neue Zeit, 29. 9. 1977; AChB, ÖNB, HS, V.117.

${ }^{26}$ Der Weg zur Scheidungsreform. „Die Sternstunde", in: Profil 24 (1978) 16.
} 
arbeitet wurde deshalb eine neue Regelung wie sei im Wesentlichen später auch Gesetz wurde. Nach dieser sollte ein nach $\S 55$ EheG aus überwiegendem Verschulden des anderen Ehegatten geschiedener Ehegatte denselben Unterhaltsanspruch wie bei aufrechter Ehe nach $\S 94$ ABGB haben. Wenn der unterhaltspflichtige Partner eine neue Ehe einging, sollte die Unterhaltspflicht für den neuen Ehegatten nur dann ins Gewicht fallen, wenn es bei Abwägung aller Gründe, besonders des Lebensalters und der Gesundheit des geschiedenen und des neuen Gatten, der Dauer ihres gemeinsamen Haushalts mit dem Verpflichteten und dem Wohl der Kinder billig erschien. ${ }^{27}$

Parteiengespräche im Sommer 1977, die zur Vorbereitung der parlamentarischen Arbeit dienen sollten, hatten mit der ÖVP hingegen zu einer weiteren Annäherung in der konkreten Ausgestaltung der einvernehmlichen Scheidung bzw. ebenfalls zur Ausarbeitung eines neuen Vorschlags geführt. Die einvernehmliche Scheidung, die nun in einem eigenen $\S 55$ a geregelt wurde, war jetzt an folgende Voraussetzungen geknüpft: Mindestdauer der Ehe ein Jahr, sechsmonatige Aufhebung der ehelichen Lebensgemeinschaft, Zugeständnis der Zerrüttung, Einvernehmen über die Scheidung sowie Vereinbarung über die Scheidungsfolgen..$^{28}$ Keine Annäherung war in diesen Gesprächen jedoch in der Befristung des Widerspruchs erreicht worden, die auch von der katholischen Kirche weiter bekämpft wurde. So wurde von der österreichischen Bischofskonferenz das Jahr 1978 nicht nur zum "Jahr der Familie“ erklärt und eine Verfassungsbestimmung zum Schutz und Förderung von Ehe und Familie gefordert, ${ }^{29}$ sondern in einer Stellungnahme vom 30. Jänner 1978 auch betont, dass die österreichischen Bi-

\footnotetext{
${ }^{27}$ LEHNER, Familie - Recht - Politik 545.

${ }^{28}$ Ebd. 539.

${ }^{29} 1978$ Jahr der Familie, in: Ehe und Familie 12 (1977)

2.
}

schöfe mit allem Nachdruck ein zu freizügiges Scheidungsrecht ablehnen. ${ }^{30}$

Die parlamentarischen Verhandlungen über das neue Scheidungsrecht begannen im März 1978, nachdem sich der Justizausschuss bereits am 16 . März 1977 zum ersten Mal mit den beiden noch offenen Regierungsvorhaben in der Familienrechtsreform, der Neuordnung des gesetzlichen Erbrechts des Ehegatten und des gesetzlichen ehelichen Güterstandes sowie der Änderung des Ehegesetzes, beschäftigt hatte. Hierbei war nicht nur die Einsetzung eines personengleichen Unterausschusses, sondern auch ein Vorziehen des Erb- und Ehegüterrechts beschlossen worden. ${ }^{31}$ Begleitet waren die Beratungen durch eine nochmalige Intensivierung des Drucks der katholischen Kirche, das neue Scheidungsrecht doch noch stärker in ihrem Sinn beeinflussen zu können. Kontaktiert wurden in diesem Sinn nicht nur Justizminister Broda und Klubobmann Heinz Fischer sondern auch Bundeskanzler Kreisky, der in gesellschaftspolitischen Fragen eher zur Vorsicht neigte und in der Partei zu jenen Vertretern zählte, die sich vor allem seit der zweiten Hälfte der 1950er Jahren um eine Öffnung gegenüber der katholischen Kirche bemüht hatte. ${ }^{32}$ Hierbei hat sich besonders der Katholische Familienverband unter seinem Obmann Helmuth Schattovits für eine stärkere Berücksichtigung seines Modells ausgesprochen und in Hinblick auf Brodas Verweis auf das Ausland vor "Zuständen wie in Schweden" gewarnt, wo seit 1974 eine Scheidung gegen den Widerstand des Partners nach einer zweijährigen Trennung möglich war. Reagiert hatte man auf die Kritik seitens der SPÖ bereits Anfang des Jahres mit einem weiteren Vorschlag zur Neuregelung des § 55 EheG. Wichtigster Punkt dieser

\footnotetext{
${ }^{30}$ Erklärung der Österreichischen Bischofskonferenz zur Scheidungsreform vom 30.1.1978. AChB, ÖNB, HS, V.123.

${ }^{31}$ EYPELTAUER, Familienrechtsreform.

32 STEGER, Brückenschlag.
} 
Modifikation war die Aufnahme der vom Katholischen Familienverband geforderten Abwägungsklausel bzw. der im bisherigen Entwurf nicht vorgesehenen richterlichen Abwägung von Härten im Hinblick auf die Scheidung. Auch diese Abwägung sollte aber nur innerhalb einer Frist - sie betrug nun sechs Jahre - nach der Auflösung der häuslichen Gemeinschaft Beachtung finden. ${ }^{33}$ Am harten Kern der Reform war somit wiederum nicht gerüttelt worden, weshalb der Katholische Familienverband auch weiterhin vor einer „Fallfrist" warnte und hier auch deutlich auf die heftigen Auseinandersetzungen rund um die Einführung der Fristenregelung hinwies - hatte deren Einführung 1973/74 doch zu einem heftigen Protest der Kirche und einem von der "Aktion Leben" lancierten Volksbegehren geführt, das bis dato das erfolgreichste Volksbegehren der Zweiten Republik war. Broda warb indessen bei Kardinal König um Verständnis für seine Reform, da „nach aller Lebenserfahrung" eine Wiederherstellung der ehelichen Lebensgemeinschaft nach sechsjähriger Auflösung der Ehe nicht mehr erwartet werden kann. Zugleich betonte er, dass man beim gewählten Zeitraum nicht nur in der europäische Mitte liege, sondern hier auch eine so weitreichende materielle Absicherung der schutzbedürftigen Ehegattin geschaffen werde, wie sie in keinem anderen Land Europas bestehe. Dass sich die SPÖ zur partnerschaftlichen Familie als Form dauernden Zusammenlebens bekenne, zeige auch das neue Parteiprogramm der SPÖ.34 Bundeskanzler Kreisky hatte sich hingegen bereits auf dem Parteitag der SPÖ im Mai 1978 hinter die Reform gestellt und festgehalten, dass man versucht habe, der katholi-

\footnotetext{
${ }^{33}$ Katholischer Familienverband Österreichs, Das neue Familienrecht 48 und 63.

${ }^{34}$ Schreiben von Christian Broda an Kardinal König vom 23. 5. 1978, in: AChB, ÖNB, HS, V.123.
}

schen Kirche so weit als möglich entgegenzukommen. ${ }^{35}$

Bei den Beratungen im Parlament konnte mit Ausnahme der zeitlichen Befristung der richterlichen Abwägung von Härten in allen Punkten Einigkeit erzielt werden. Hierbei ist es in der Frage, ob es notwendig wäre, die einvernehmliche Scheidung an Fristen zu binden, nochmals zu einer umfangreichen Diskussion gekommen, die schließlich damit abgeschlossen wurde, auf die Mindestdauer der Ehe $\mathrm{zu}$ verzichten, das Erfordernis der sechsmonatigen Aufhebung der ehelichen Lebensgemeinschaft jedoch beizubehalten. Gleichfalls wurde als flankierende Maßnahme beschlossen, dem Gericht die Möglichkeit zu geben, das Verfahren von Amts wegen auf maximal sechs Monate zu unterbrechen, wenn es zur Überzeugung gelangt, dass eine Aussicht auf Wiederherstellung der ehelichen Lebensgemeinsacht besteht. In der Frage der Sechs-Jahres-Frist, die die ÖVP nicht nur mit dem Hinweis auf negative Rückwirkungen auf die Ehegesinnung ${ }^{36}$ sondern auch mit dem von ÖVP-Obmann Josef Taus geprägten Schlagwort der „Wegwerffrau“ ablehnte, konnte jedoch nur mit der FPÖ Konsens erzielt werden. Diese hatte sich bereits in ihren Zielsetzungen zur Familienrechtsreform vom 17. Oktober 1977 am stärksten von allen Parlamentsparteien für das Zerrüttungsprinzip ausgesprochen. ${ }^{37}$

In Folge wurde, um nicht die ganze Scheidungsreform einer einstimmigen Beschlussfassung zu opfern, eine Teilung der Scheidungsreform beschlossen. D.h. wenn schon keine vollständige Einigung in der Sache erzielt worden war, wurde zumindest eine amikale Lösung beim Ab-

${ }^{35}$ SPÖ (Hg.), Protokoll des 24. ordentlichen Bundesparteitags der SPÖ vom 18.-20. 5. 1978 in Wien (Wien 1978) 148.

${ }^{36}$ Scheidung: SPÖ sagt 6 statt 5. ÖVP weiter gegen fixe Frist, in: Kleine Zeitung, 5. 4. 1978.

${ }^{37}$ Freiheitliche Zielsetzungen zur Familienrechtsreform vom 17. 10. 1977, in: LEHNER, Familie - Recht Politik 593. 
stimmungsmodus gefunden. So umfasste ein Antrag des Justizausschusses die neuen Bestimmungen über das Ehegattenerb- und Ehegüterrecht sowie jene Teile der Scheidungsreform, über die man sich auch mit der ÖVP geeinigt hatte, während ein zweiter Antrag die Neuregelung des Widerspruchs regelte. ${ }^{38}$ Die Beschlussfassung im Parlament brachte am 15. Juni 1978 eine einstimmige Annahme des neuen Ehegattenerbrechts, des Ehegüterrechts und der einvernehmlichen Scheidung. Gleichfalls einstimmig wurden auch die neuen Scheidungsfolgen bei einer Scheidung nach $\S 55$ EheG beschlossen - inklusive der damit verbundenen sozialversicherungsrechtlichen Neuerungen, die im Kern den 1976 vorgeschlagenen Änderungen entsprachen, aber um die Einbeziehung der Krankenversicherung erweiterten worden waren. ${ }^{39}$ Die Bestimmung in $\S 55$ Abs. 3 EheG, wonach dem Scheidungsbegehren in jedem Fall stattzugeben sei, wenn die häusliche Gemeinschaft seit sechs Jahren aufgelöst ist, wurde bei namentlicher Abstimmung jedoch nur mit den Stimmen von SPÖ und FPÖ beschlossen und von ÖVPJustizsprecher Hauser in der Debatte im Plenum des Nationalrats dahingehend kommentiert, dass die ÖVP eine Schlacht an der "MaginotLinie des Rechts" verloren hätte.40 In Folge musste sie, nachdem das neue Gesetz im ÖVP dominierten Bundesrat beeinsprucht worden war, am 30. Juni 1978 im Nationalrat mittels eines Beharrungsbeschlusses bestätigt werden. In Kraft getreten sind beide Gesetze mit 1. Juli 1978, womit nicht nur die Familienrechts-, sondern auch die Scheidungsreform der 1970er Jahre abgeschlossen war. Weitere Reformen wurden in diesen Jahren nicht mehr angestrebt, wenn auch zu Beginn der Diskussion noch Überlegungen angestellt worden waren, das

\footnotetext{
38 Ebd. 541f.

39 MeISEL, Scheidungsreform.

${ }^{40}$ Scheidung trennt die Einigkeit, in: Kurier, 16. 6. 1978 .
}

Scheidungsrecht insgesamt einer größeren Revision zu unterziehen.

\section{Reaktionen}

Aufgenommen wurde das neue Scheidungsrecht - wie bereits die vorhergehende Diskussion vermuten lässt - gespalten. So war von einzelnen Vertretern der ÖVP noch im Wahlkampf 1983 zu hören, dass die „Fristenautomatik“ bei der Scheidung unter einer ÖVP-Regierung fallen werde, ${ }^{41}$ und auch Kardinal König sowie der Katholische Familienverband protestierten nach der Beschlussfassung gegen das neue Scheidungsrecht. ${ }^{42} \mathrm{Zu}$ einem neuerlichen Kulturkampf wie in der Frage der Fristenregelung kam es indessen nicht, wenn auch in der Diskussion mehrfach warnend und drohend zugleich auf diese verwiesen worden war. Das Verhältnis zwischen SPÖ und katholischer Kirche wurde nach der Einführung der Fristenregelung durch die Scheidungsreform zwar erneut auf eine harte Probe gestellt. Maßgeblich dadurch bedingt, dass der Dialog zwischen SPÖ und katholischer Kirche trotz der Konflikte in der Rechtsreform nicht abriss und hier auch immer wieder die Bereitschaft zur Verständigung betont wurde, konnte der Prozess der Aussöhnung zwischen Partei und katholischer Kirche jedoch fortgesetzt werden.

Von Seiten der Fachwelt wurde das neue Scheidungsrecht zwar grundsätzlich anerkannt, aber auch darauf verwiesen, dass dieses eine Ungleichbehandlung gegenüber den ebenfalls schuldlos, aber nach anderen Tatbeständen Geschiedenen bringen würde - hatte man sich im Zuge der Reformdiskussion Seitens des Justizministeriums doch dafür entschlossen, nur das

\footnotetext{
${ }^{41}$ FIEDLER, Auswirkungen der Familienrechtsreform 433.

${ }^{42}$ Kardinal: „Scheidungsgesetze ein Affront gegen die Kirche“, in: Die Presse, 28. 6. 1978.
} 
Scheidungsfolgenrecht bei einer Scheidung nach $\S 55$ EheG zu verbessern. Gewarnt wurde in diesem Sinn sogar davor, dass das neue Scheidungsrecht ein neues Problem der "Papierehen“ hervorbringe würde, da man sich in Zukunft gut überlegen müsse, die Scheidung wegen eines schuldhaften Verhaltens des Ehepartners selbst einzubringen oder so lange zu warten, bis der andere Ehegatte dies tat. ${ }^{43}$

Heute wird in der Reform der 1970er Jahren eine weitere Stärkung des Zerrüttungsprinzips gesehen, wobei vor allem die Bedeutung der einvernehmlichen Scheidung betont wird. ${ }^{44}$ Wie die historische Entwicklung zeigt, war diese jedoch keineswegs der Ausgangs- oder Mittelpunkt der Reform, und vor allem hat sie auch nicht jene Auseinandersetzung ausgelöst, wie es die Reform des $\S 55$ EheG tat.

\section{Abkürzungen:}

AChB Archiv Christian Broda

ÖNB Österreichische Nationalbibliothek

HS Handschriftensammlung

Siehe auch das allgemeine Abkürzungsverzeichnis: http://www.rechtsgeschichte.at/beitraege/abk.pdf

\section{Literatur:}

1978 Jahr der Familie, in: Ehe und Familie 12 (1977) 2.

Christian BrodA, Ein „offenes“ Programm, in: Die Zukunft 8 (1970) 18-20.

Bundesministerium für Justiz, Familienrechtsreform konkret (Wien ${ }^{31975)}$.

Bundesministerium für Justiz, Scheidungsreform konkret (Wien 1976).

Bundesministerium für Justiz, Scheidungsreform konkret (Wien ${ }^{2}$ 1976).

Doris EYPELTAUER, Die Familienrechtsreform im parlamentarischen Willensbildungsprozess (phil. Diss., Univ. Wien 1981).

Franz FIEDLER, Auswirkungen der Familienrechtsreform, in: Andreas KHOL, Alfred STIRNEMANN

\footnotetext{
${ }^{43}$ KREJCI, Neues Scheidungsrecht.

${ }^{44}$ FlossmanN, Österreichische Privatrechtsgeschichte 92.
}

(Hgg.), Österreichisches Jahrbuch für Politik 1983 (Wien-München 1984) 417-450.

Ursula FlossmanN, Österreichische Privatrechtsgeschichte (Wien-New York ${ }^{6} 2008$ ).

Maximilian GotTsChlich, Oswald PANAGL, Manfried WELAN, Was die Kanzler sagten. Regierungserklärungen der Zweiten Republik 1945-1987 (=Studien zu Politik und Verwaltung 15, WienKöln 1989).

Lothar GRUCHMANN, Das Ehegesetz vom 6. Juli 1938, in: ZNR 11 (1989) 63-83.

Ulrike HARMAT, Ehe auf Widerruf? Der Konflikt um das Eherecht in Österreich 1918-1938 (Frankfurt am Main 1999).

Katholischer Familienverband Österreichs (Hg.), Das neue Familienrecht. Eine Informationsschrift des Katholischen Familienverbandes Österreich (= Brennpunkt Familie 2, Wien 1978).

Heinz KREJCI, Neues Scheidungsrecht und soziale Sicherung, in: Juristische Blätter 7/8 (1979) 169182.

Oskar LEHNER, Familie - Recht - Politik. Die Entwicklung des österreichischen Familienrechts im 19. und 20. Jahrhundert (= Linzer Universitätsschriften, Monographien 13, Wien-New York 1987).

Mehr Rechtsschutz für den Staatsbürger. Justizprogramm für 1970-1974. Beschlossen vom Parteirat der SPÖ am 22. November 1969.

Wilhelm MEISEL, Die Scheidungsreform und ihre Auswirkung im Sozialversicherungsrecht, in: Oswin MARTINEK, Josef CZERNY, Josef WEIDENHOLZER (Hgg.), Arbeitswelt und Sozialstaat. Festschrift für Gerhard Weißenberg (Wien-München-Zürich 1980) 373-383.

Österreichisches Anwaltsblatt, Sondernummer, November 1972.

Martin Peter ScHAUER, Das Recht der Ehescheidung im 19. und 20. Jahrhundert. Vom Verschuldensprinzip zum Zerrüttungsprinzip (jur. Diss., Univ. Linz 1998).

Martin SCHAUER, Die "gesunde Nachkommenschaft" in der NS-Zeit bis heute. Ein Beitrag zum österreichischen Scheidungsrecht, in: Ursula FLOSSMANN (Hg.), Nationalsozialistische Spuren im Recht. Ausgewählte Stolpersteine für ein selbstbestimmtes Frauenleben (= Linzer Schriften zur Frauenforschung 12, Linz 1999) 175-125.

SPÖ (Hg.), Protokoll des 24. ordentlichen Bundesparteitags der SPÖ vom 18.-20. Mai 1978 in Wien (Wien 1978).

Gerhard STEGER, Der Brückenschlag. Katholische Kirche und Sozialdemokratie in Österreich (Wien 1982. 